\title{
Epidemiology of sepsis in Korea: a population-based study of incidence, mortality, cost and risk factors for death in sepsis
}

\author{
Joonghee Kim¹, Kyuseok Kim², Heeyoung Lee ${ }^{3}$, Soyeon Ahn ${ }^{4}$ \\ 'Department of Emergency Medicine, Seoul National University Bundang Hospital, Seongnam, Korea \\ ${ }^{2}$ Department of Emergency Medicine, Seoul National University College of Medicine, Seoul, Korea \\ ${ }^{3}$ Department of Epidemiology, Seoul National University Bundang Hospital, Seongnam, Korea \\ ${ }^{4}$ Medical Research Collaborating Center, Seoul National University Bundang Hospital, Seongnam, Korea
}

Objective To investigate the epidemiology of sepsis in Korea and identify risk factors for death in sepsis.

Methods We conducted a longitudinal, population-based epidemiological study of sepsis in Korea from 2005 to 2012 using the National Health Insurance Service-National Sample Cohort, a population-based cohort representing $2.2 \%$ of the Korean population. The primary objective was to assess the incidence, mortality and cost of sepsis. The secondary objective was to identify the risk factors for death in sepsis. Claim records of admitted adult patients (aged $\geq 15$ years) were analyzed. Sepsis was defined as 1) bacterial or fungal infection or the conditions they often complicate, 2) prescription of intravenous antibiotics, and 3) presence of any organ dysfunction. Comorbidities were defined using the Charlson/Deyo method. Risk factors for 6-month mortality were assessed using multivariable logistic regression.

Results A total of 22,882 cases were identified. Both incidence and 6-month mortality increased from 265.7 (95\% confidence interval [Cl], 254.7 to 277.1$)$ to 453.1 (95\% Cl, 439.0 to 467.5 ) per $100,000$ person-years (P-trend $<0.001)$ and from $26.5 \%(95 \% \mathrm{Cl}, 24.4 \%$ to $28.8 \%)$ to $30.1 \%$ $(95 \% \mathrm{Cl}, 28.4 \%$ to $31.9 \%)$, respectively. After standardization, the increasing trend of incidence was slower but still significant ( $\mathrm{P}$-trend $<0.001)$, while that for mortality was not ( $\mathrm{P}$-trend 0.883 ). The average cost increased by $75.5 \%$ ( $\mathrm{P}$-trend $<0.001)$. Multivariable logistic regression identified various risk factors for mortality.

Conclusion The burden of sepsis in Korea was high and is expected to increase considering the aging population. Proactive measures to curtail this increase should be sought and implemented.

Keywords Sepsis; Epidemiology; Incidence; Mortality; Risk factors

Capsule
$\begin{aligned} & \text { What is already known } \\ & \text { The incidence of sepsis and mortality have increased worldwide. The epidemio- } \\ & \text { logical characteristics of sepsis in Korea are yet unknown. }\end{aligned}$
$\begin{aligned} & \text { What is new in the current study } \\ & \text { Although the incidence of sepsis in Korea has increased, the mortality rate has } \\ & \text { remained steady. }\end{aligned}$

eISSN: 2383-4625

Received: 30 January 2018

Revised: 11 April 2018

Accepted: 26 June 2018

Correspondence to: Kyuseok Kim Department of Emergency Medicine, Seoul National University College of Medicine, 103 Daehak-ro, Jongno-gu, Seoul 03080, Korea

E-mail:dreinstein70@gmail.com ORCID

https://orcid.org/0000-0002-7991-9428

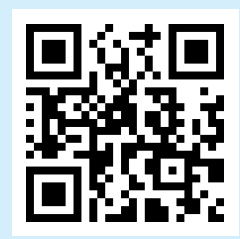

How to cite this article:

Kim J, Kim K, Lee H, Ahn S. Epidemiology of sepsis in Korea: a population-based study of incidence, mortality, cost and risk factors for death in sepsis. Clin Exp Emerg Med 2019;6(1):49-63.

This is an Open Access article distributed under the terms of the Creative Commons Attribution Non-Commercial License (http:// creativecommons.org/licenses/by-nc/4.0/). 


\section{INTRODUCTION}

Sepsis is defined as life-threatening organ dysfunction caused by a dysregulated host response to infection. It has very high morbidity and mortality rates and is a public health problem worldwide. $^{1,2}$

To improve its outcomes, it is critical to provide optimal management in every aspect of care including initial screening, hemodynamic optimization, antibiotic treatment, and source control. ${ }^{3}$ This requires rapid but coordinated approaches from multiple disciplines including the general public, emergency medical service, and hospitals with the support of proactive governmental policies. In this process, detailed national epidemiologic information about sepsis can play a critical role. However, no nationwide population-based study has been conducted in Korea compared to many other developed countries where such epidemiological information has been used to support healthcare policy making. ${ }^{4-20}$

We conducted a longitudinal, population-based epidemiological study of sepsis in Korea using a nationally representative cohort database. The primary objective of the study was to estimate the incidence, mortality and cost of sepsis in Korea. The secondary objective was to identify risk factors for death due to sepsis in the Korean population.

\section{METHODS}

This study was approved by IRB of Seoul National Bundang Hospital, institutional review board determined the study was exempt (SNUBH IRB X-1502-288-903).

\section{Data source}

The data source was the National Health Insurance Service-National Sample Cohort, a population-based cohort established by the Korean National Health Insurance Service. ${ }^{21}$ It contains claim information of over one million individuals (2.2\%) who were ran- domly sampled from almost the entire Korean population using stratification. It provides information about diagnoses, prescriptions, procedures, and related costs as well as basic demographic information such as age, sex, and socioeconomic status. Diagnoses are coded using the Korean Classification of Diseases, sixth revision which is based on the tenth revision of the International Statistical Classification of Diseases and Related Health Problems (ICD-10). It also contains information about disability and death based on the disability registration data and the officially-issued death certificates, respectively. We used its most recent release which contains claim data from 2005 to 2012. The detailed description of the cohort can be found in a previous paper. ${ }^{21}$

\section{Case selection and the variables analyzed}

Claim records of adult patients (aged $\geq 15$ years) admitted to hospitals were used for case definition. We identified sepsis using the following procedures (Fig. 1). The entire claim information for an admission is often separated into multiple records covering different time segments of admission. Therefore, we first linked successive claim records corresponding to each admission. Then a case of sepsis was defined as an admission with at least one occurrence of claim record fulfilling all the following conditions: 1) bacterial or fungal infection or the conditions that are often complicated by such infections (e.g., surgical abdomen) as indicated by the primary or first secondary diagnostic codes (please refer to the Appendix 1 for a detailed list of ICD-10 codes), 2) prescription of intravenous antibiotics (parenteral antibiotics with Anatomical Therapeutic Chemical Classification System code J01), and 3) presence of organ dysfunction involving at least one system (please refer to the Appendix 1 for a detailed list of ICD-10 codes). Septic shock was defined as the inclusion of R57.2 (septic shock) in the diagnostic codes or the use of intravenous vasopressors such as dopamine, dobutamine, norepinephrine, vasopressin, and epinephrine.

Comorbidities for each of the cases were defined using the first claim record fulfilling the sepsis criteria and the admission claim

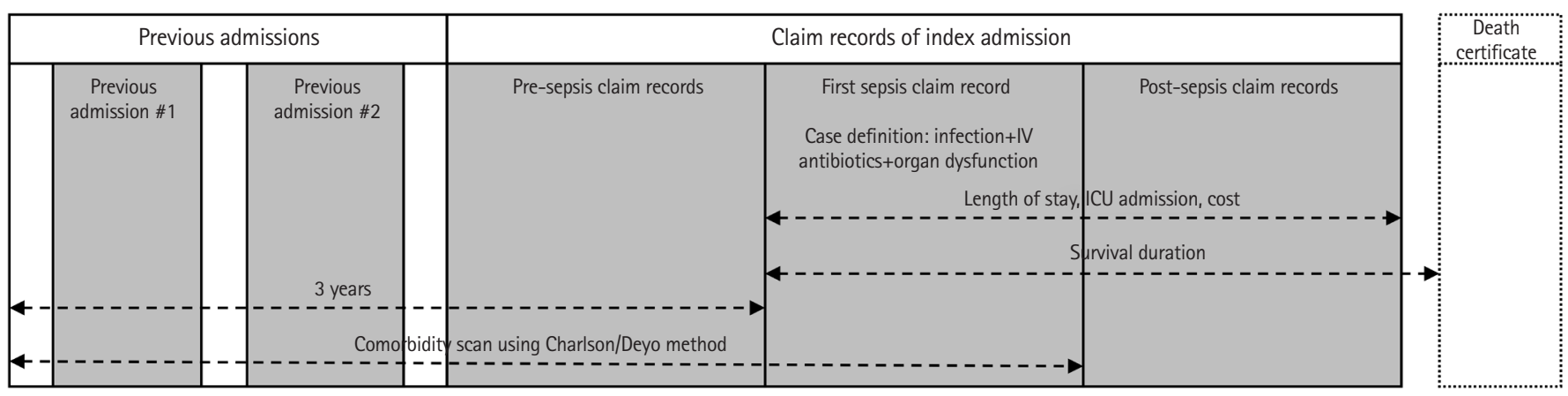

Fig. 1. An illustration of the case identification and the assessment of variables. IV, intravenous; ICU, intensive care unit. 


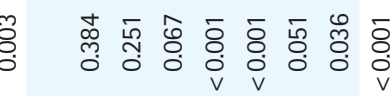

웅

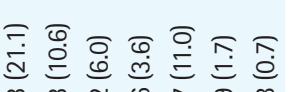

更

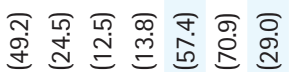

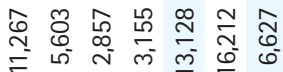

ㅈ. $\bar{\sigma} F$

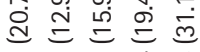

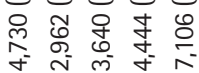

$\bar{\delta}$
0
v

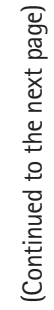

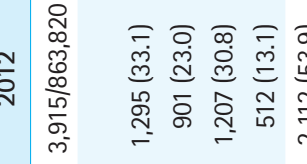

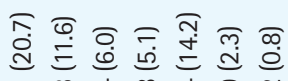

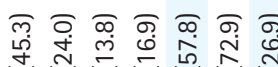

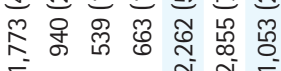

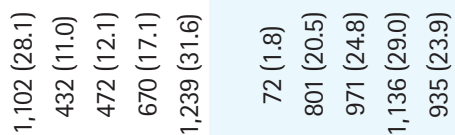

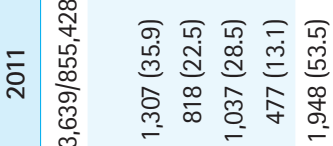

बृ

व.

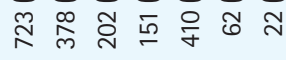

品 \&

สำ $\overline{0} \bar{m}$

엇 종

व્فं

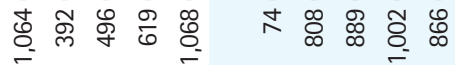

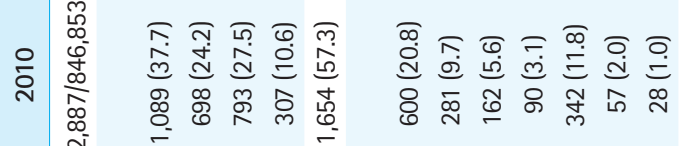

สู.

于 $\bar{\sigma} \overline{\mathrm{g}}$

โก โุ

$\stackrel{=}{=} \equiv=$ ल

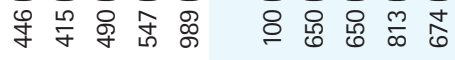

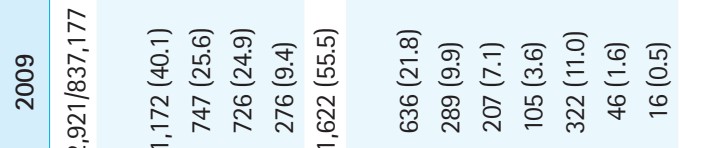

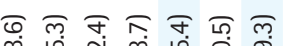

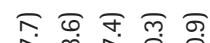

ซ $\bar{~}$

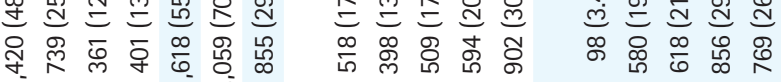

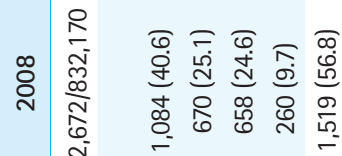

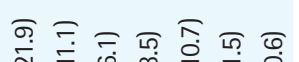

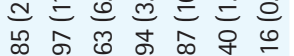

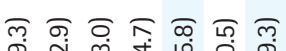

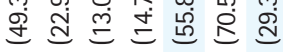

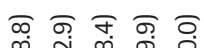

ল

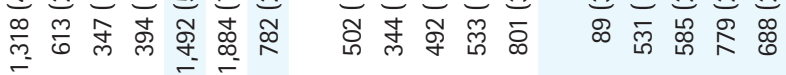

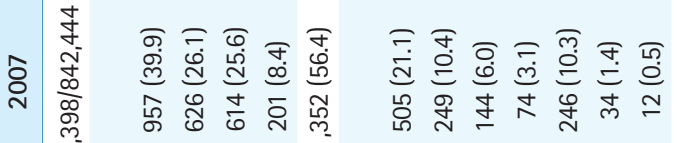

우 ㅎำ

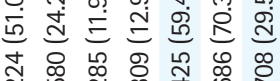

ढ़ बृ

क $\bar{\circ} \bar{\sigma}$

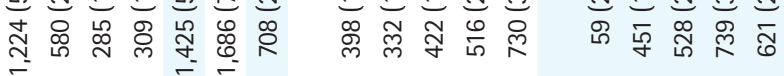

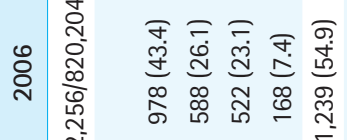

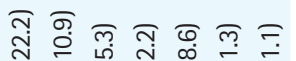

F $\sigma \bar{\sigma} \sigma \bar{\sigma}$

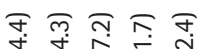

ㄸำ

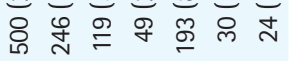

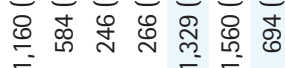

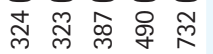

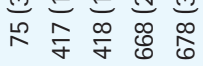

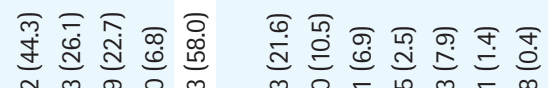

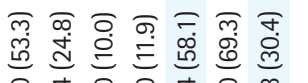

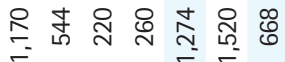

두 $\bar{\sigma} \overline{0}$

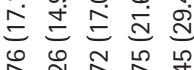

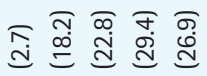
命命字郘 孚岱占品点

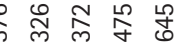

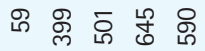

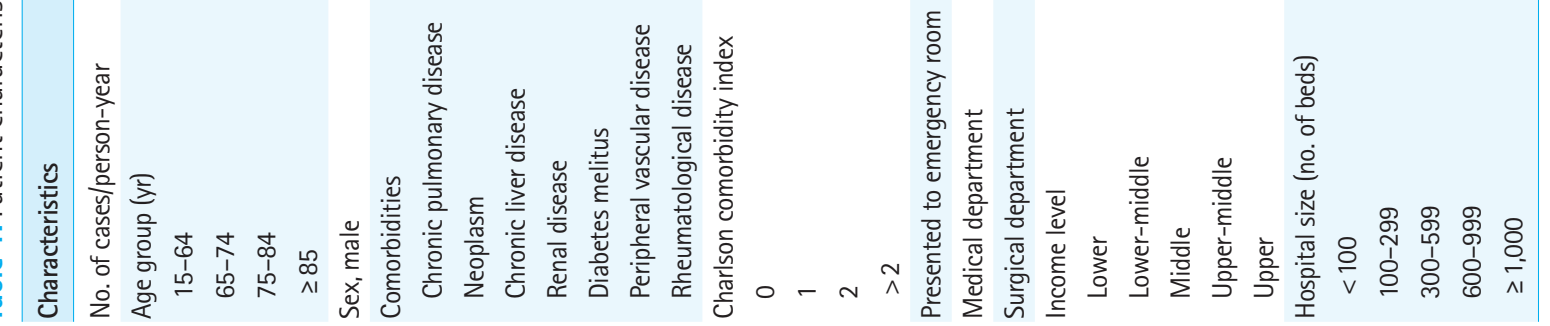


帝

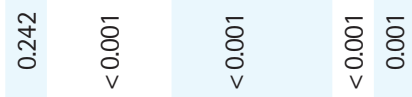

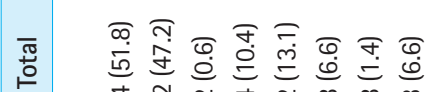

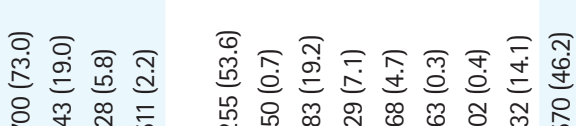

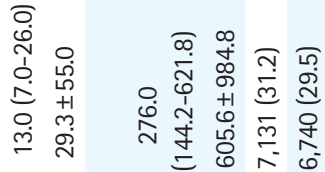

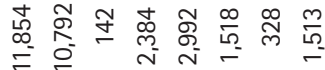

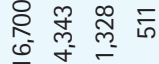

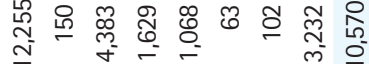

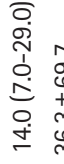

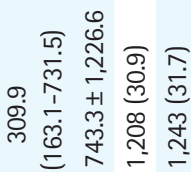

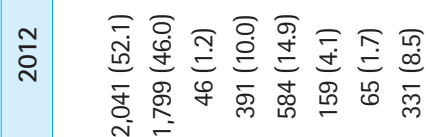

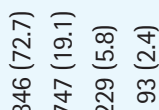

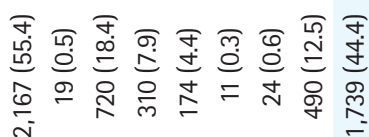

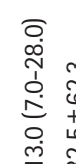

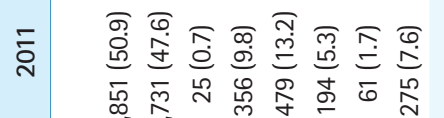

₹

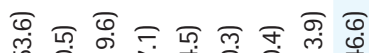

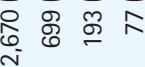

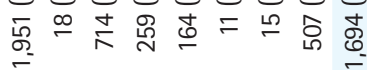

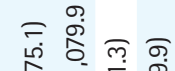

0.

$=$ =

은

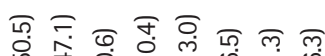

雪 $\overline{0}$

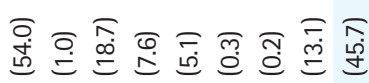

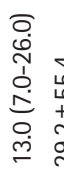

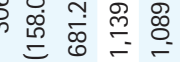

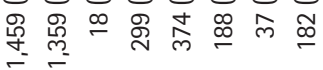

究的究

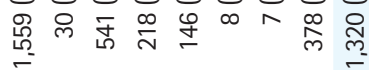

雾

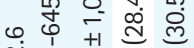

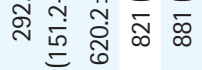

ঃั่

זิ

สุ $\check{(ต) ~}$

लํ

울

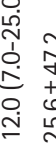

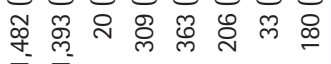

旁品点

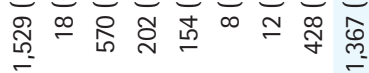

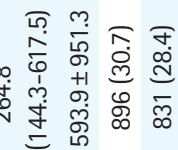

芩

ซ

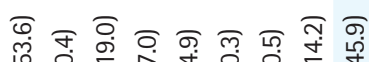

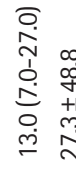

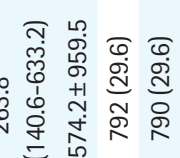

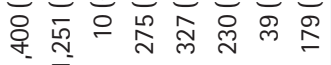

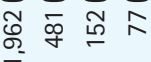

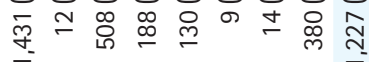

뭉

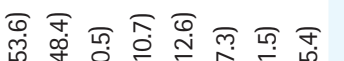

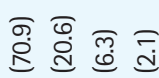

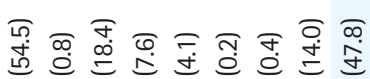

宅

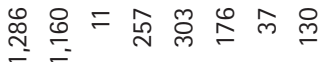

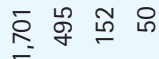

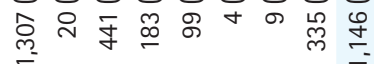

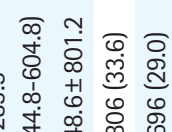

守兽

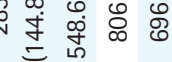

Oें

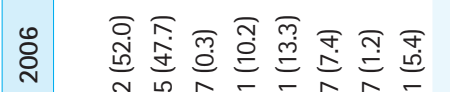

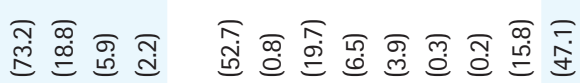

我

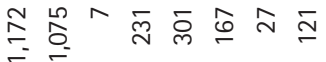

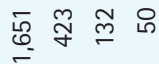

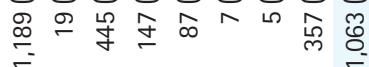

雨等

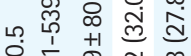

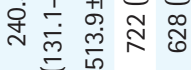

중 중

종

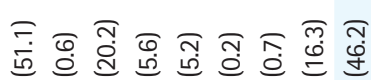

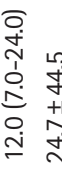

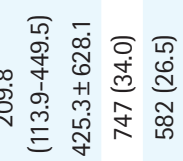

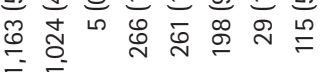

总 总范品

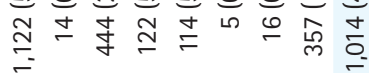

ì

ن $\frac{\pi}{\frac{\pi}{2}}$

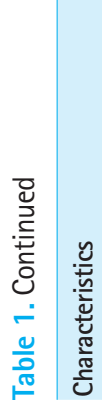

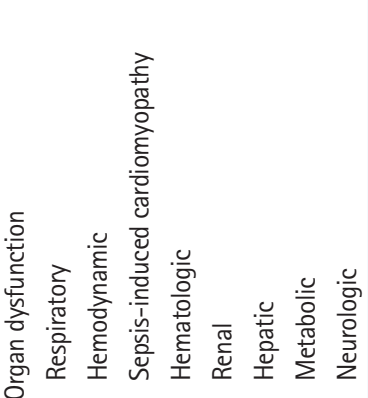
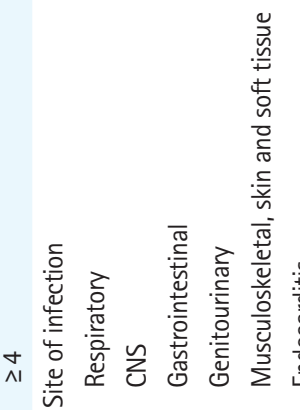

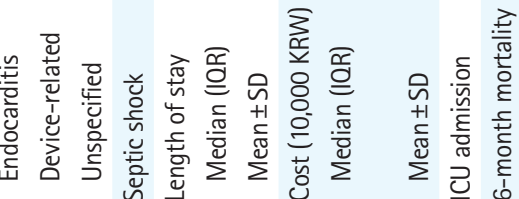

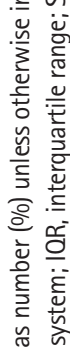

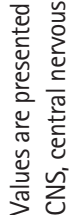


records within three years prior to the first sepsis record using the Charlson/Deyo method. ${ }^{22-24}$ Ancillary variables including household income level, emergency room (ER) presentation, type of admitting department, and hospital capacity measured by the number of beds were also included in the analyses. Length of stay, overall cost, intensive care unit (ICU) admission and 6-month mortality were included as outcome measures. We excluded the claim records beginning more than a year after the event when calculating the length of stay and cost because we thought prolonged admission more than a year after the index event would hardly be due to the index event. The detailed coding schemes are available in the Appendix 1.

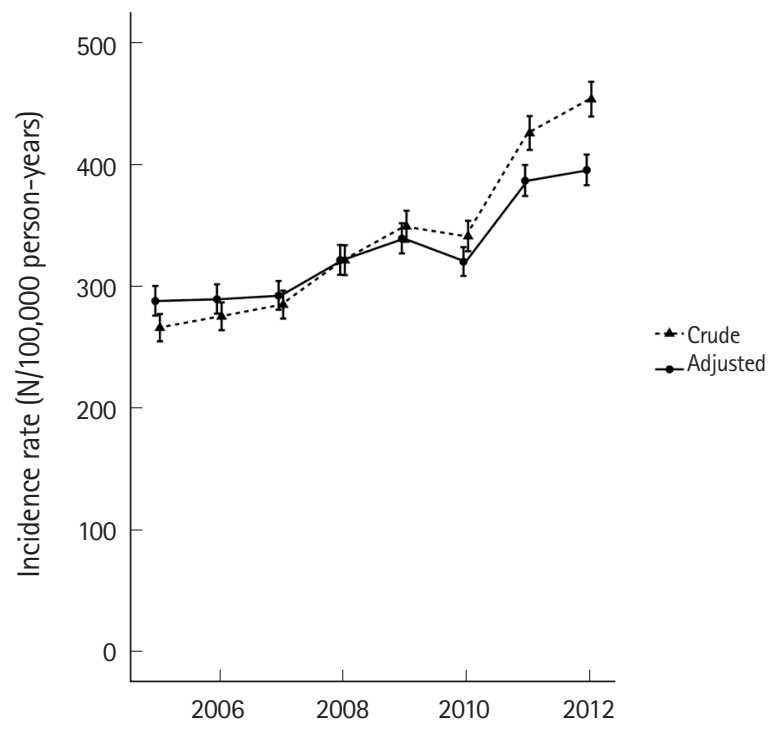

A

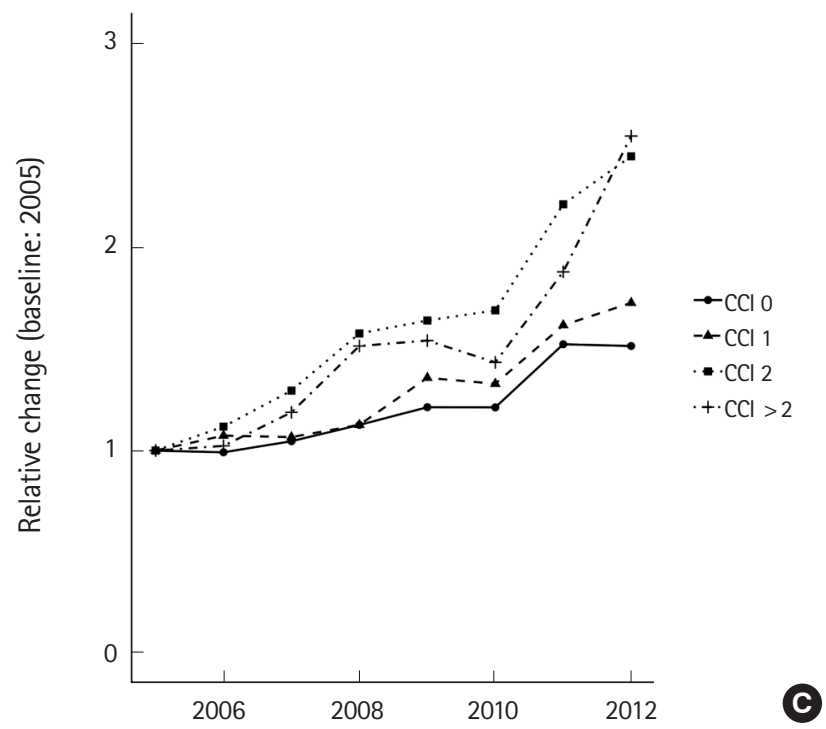

\section{Statistical analysis}

Both crude and standardized annual incidence of sepsis in the adult population (aged $\geq 15$ years) were calculated. The standardized annual incidence based on the sex-age distribution of the 2010 Korean census was estimated by direct standardization. ${ }^{25}$ Similarly, both crude and standardized 6-month mortality were calculated using the direct standardization method based on the sex-age distribution sepsis cases for the year 2010. The average (both mean and median) cost per case and the total national cost for each year were estimated in Korean won (KRW). The standardized annual average cost based on the sex-age distribution of sepsis cases from 2010 was also calculated. The trend analyses for dichotomous events were conducted using logistic regression,
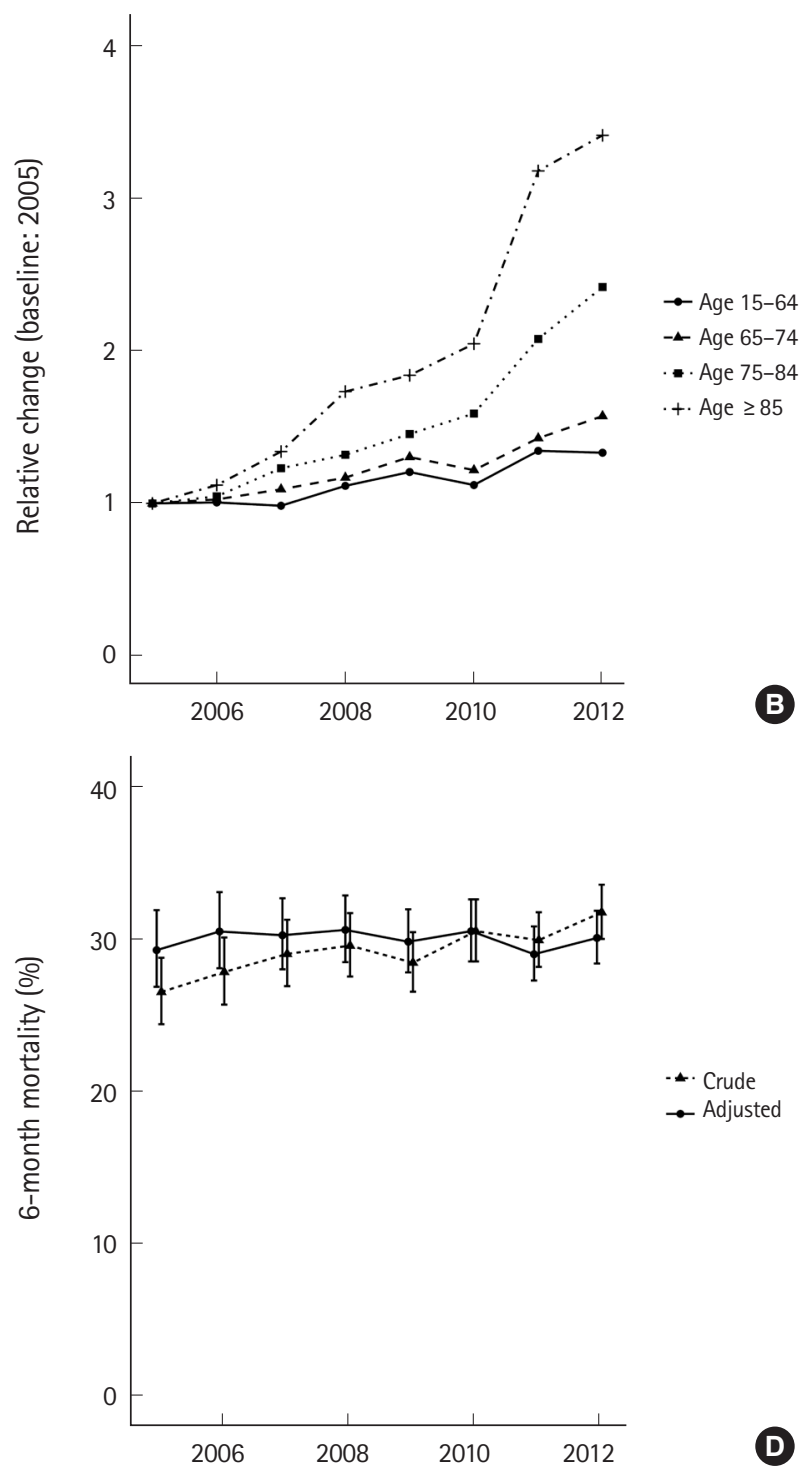

Fig. 2. Eight-year trend of sepsis incidence and mortality. (A) Crude and adjusted incidence rate of sepsis. (B) Relative change of incidence of sepsis according to the age group. (C) Relative change of incidence of sepsis according to the comorbidity. (D) Crude and adjusted 6-month mortality of sepsis. $\mathrm{CCl}$, Charlson comorbidity index. 
and those for continuous variables were conducted using linear regression.

Survival curves for the sepsis patients during the first six months were plotted using the Kaplan-Meier method and comparisons between groups were made using the log-rank test. To identify independent risk factors for mortality, we performed multivariable logistic regression analyses using two different model-building schemes. The first involved comprehensive modeling using all available variables and data. The second involved parsimonious modeling with a variable selection scheme based on Bayesian information criterion using 80\% of randomly selected cases (training set) which was later tested using the remaining $20 \%$ of cases (test set). The results of the logistic regression were presented as odds ratios and their 95\% confidence intervals (Cls). All data handling and statistical analyses were performed using $R$ ver. 3.3.2 ( $R$ Foundation for Statistical Computing, Vienna, Austria).

\section{RESULTS}

From 2005 to 2012, a total of 22,882 sepsis cases were identified from the cohort (Table 1). More than half of the patients $(14,028$; $61.3 \%$ ) were older than 65 years and had at least one comorbidity $(11,615 ; 50.8 \%)$. Over half of the cases initially presented to the $\operatorname{ER}(13,128 ; 57.4 \%)$ and over two-thirds were treated in the medical department $(16,212 ; 70.9 \%)$. Their income levels showed a polarized pattern with almost half of the cases $(11,836 ; 51.7 \%)$ in the lowest and highest income classes. Majority of the cases $(17,619 ; 77.0 \%)$ were treated in larger hospitals with capacities of 300 beds or higher. The most common type of organ dysfunc- tion was respiratory failure $(11,854 ; 51.8 \%)$ followed by hemodynamic failure $(10,792 ; 47.2 \%)$ and the most common site of infection was the respiratory system $(12,255 ; 53.6 \%)$. Almost half of the cases were complicated by septic shock $(10,570 ; 46.2 \%)$ and almost one-third of the cases involved ICU admission $(7,131$; $31.2 \%$ ). The median length of stay was 13 (interquartile range, 7 to 26) days and the median cost was 276 (interquartile range, 144.2 to 621.8$) \times 10,000 \mathrm{KRW}$ and the 6 -month mortality rate was $29.5 \%$.

Fig. 2 shows the eight-year trend of sepsis incidence and mortality. There was a significant increase in crude incidence rate of sepsis from 265.7 (254.7 to 277.1) per 100,000 person-years in 2005 to 453.1 (439.0 to 467.5) per 100,000 person-years in 2012 (P-trend < 0.001). After standardization for both age and sex, the increase was less remarkable but still significant with 287.7 (275.8 to 300.1) in 2005 to 395.1 (382.7 to 407.8) in 2012 (P-trend < 0.001). The increase was more remarkable in the elderly population and the patients with significant comorbidity burdens. The crude mortality rate also gradually increased from $26.5 \%$ (24.4\% to $28.8 \%$ ) to $30.1 \%(28.4 \%$ to $31.9 \%)$ during the period (P-trend $<0.001)$. However, the trend was not significant after sex-age standardization (from $29.3 \%$ [95\% Cl, 26.9\% to $31.9 \%$ ] to 30.5 [95\% Cl, $28.5 \%$ to $32.6 \%]$; P-trend $=0.883$ ). The crude mortality rate among patients with septic shock also gradually increased from $32.2 \%$ (28.8\% to $35.9 \%)$ to $38.5 \%$ (35.6\% to $41.5 \%)$ during the period (P-trend <0.001, Supplementary Fig. 1). However, the trend was not significant after sex-age standardization (from 36.8\% [95\% $\mathrm{Cl}, 32.8 \%$ to $41.8 \%$ ] to 36.9 [95\% Cl, 34.1\% to $39.9 \%$ ]; P-trend 0.564).

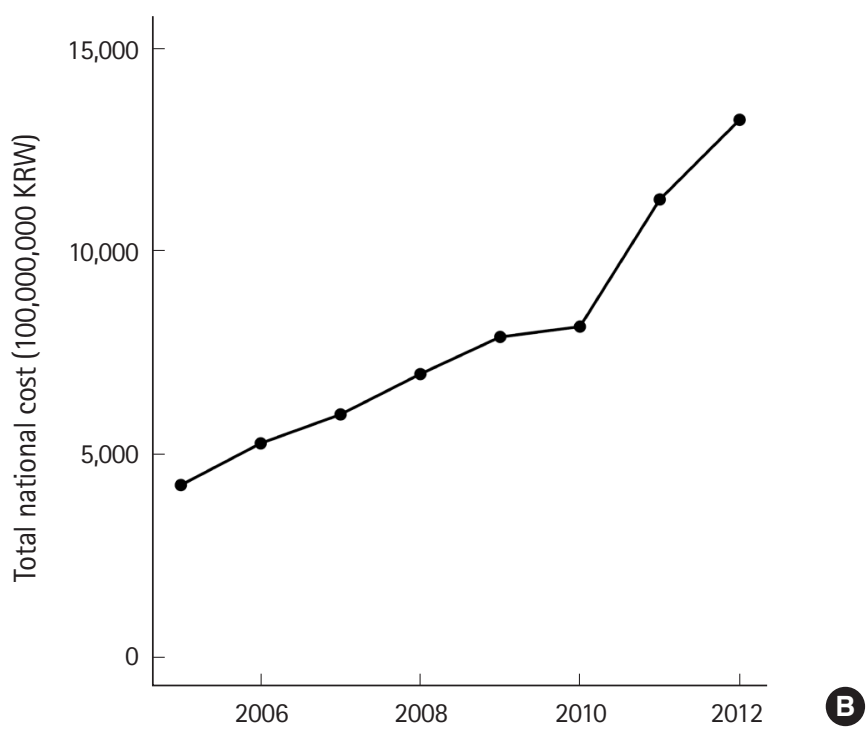

Fig. 3. Eight-year trend of national expenditure on sepsis. (A) Mean cost per case of sepsis. (B) Total national cost of sepsis. KRW, Korean won. 

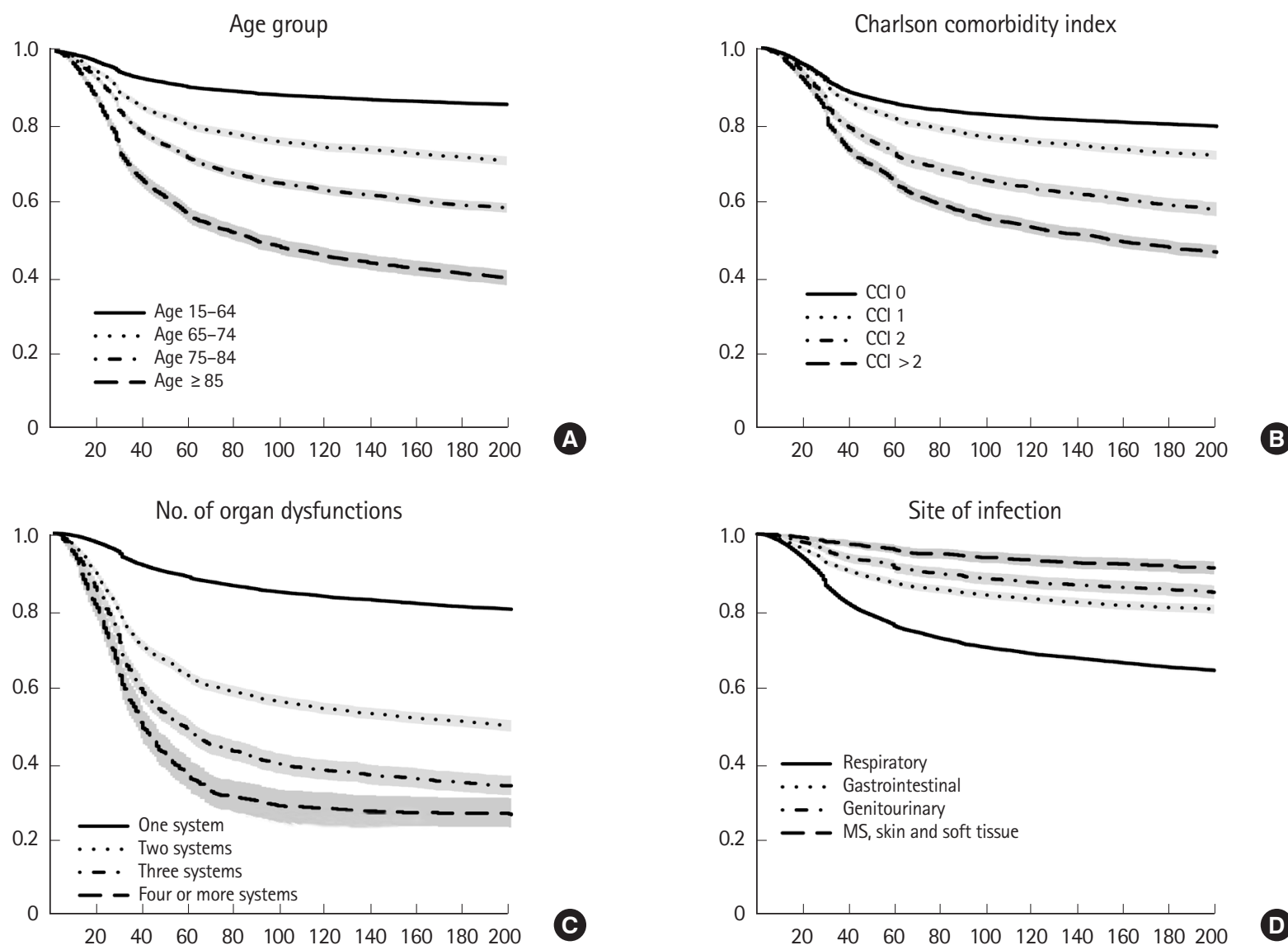

Fig. 4. Differential survival probability by age, comorbidity burden, organ dysfunction, and the site of infection. (A) Survival according to the age group. (B) Survival according to the comorbidity. (C) Survival according to the number of organ dysfunction. (D) Survival according to the site of infection. CCl, Charlson comorbidity index; MS, musculoskeletal.

The standardized average cost per case increased by $75.5 \%$ from $422.5(95 \% \mathrm{Cl}, 396.9$ to 448.1$) \times 10,000 \mathrm{KRW}$ in 2005 to $741.7(95 \% \mathrm{Cl}, 701.5$ to 781.9$) \times 10,000 \mathrm{KRW}$ in 2012 (P-trend $<0.001$ ) (Fig. 3). The estimated total national expenditure on sepsis was tripled (311.8\%) during the same period with the estimated total amount of expenditure at $13,226.5 \times 100,000,000 \mathrm{KRW}$ in 2012 (P-trend < 0.001).

Fig. 4 shows the differential survival probabilities by age, comorbidity burden, organ dysfunction and site of infection. Older age, higher comorbidity burden, multiple organ dysfunction and primary respiratory infection were associated with higher mortality rates through the first 6 months $(P<0.001$ for all). To identify independent risk factors for 6-month mortality, we performed multivariable logistic regression using two different model-building schemes. The first, using all predictors and cases, showed that older age, higher comorbidity burden, ER presentation, admission to the medical department, multiple organ dysfunction, central nervous system or unspecified site of infection (vs. respiratory system), and the development of septic shock were independently associated with increased mortality (Table 2). In contrast, female sex; higher income level; larger hospital capacity; and gastrointestinal, genitourinary, musculoskeletal, skin and soft tissue and device-related infections were associated with lower mortality. The second model was developed using $80 \%$ of the data (training set) using Bayesian information criterion for variable selection showed similar results, with patient age, sex, comorbidity level, admitting department, hospital capacity, organ dysfunction, site of infection, and the development of septic shock identified as independent predictors (Table 3 and Fig. 5). Its performance in predicting the 6-month mortality was tested in the remaining 20\% of data (test set) which yielded an area under the receiver operating characteristic curve of 0.840 (95\% Cl, 0.829 to 0.852$)$ (Fig. 6).

\section{DISCUSSION}

This study is the first longitudinal, population-based study of sepsis in Korea. We found that sepsis is a relatively common condition with very high mortality in Korea as in other countries. Its 
Table 2. Multivariable logistic regression model for 6-month mortality

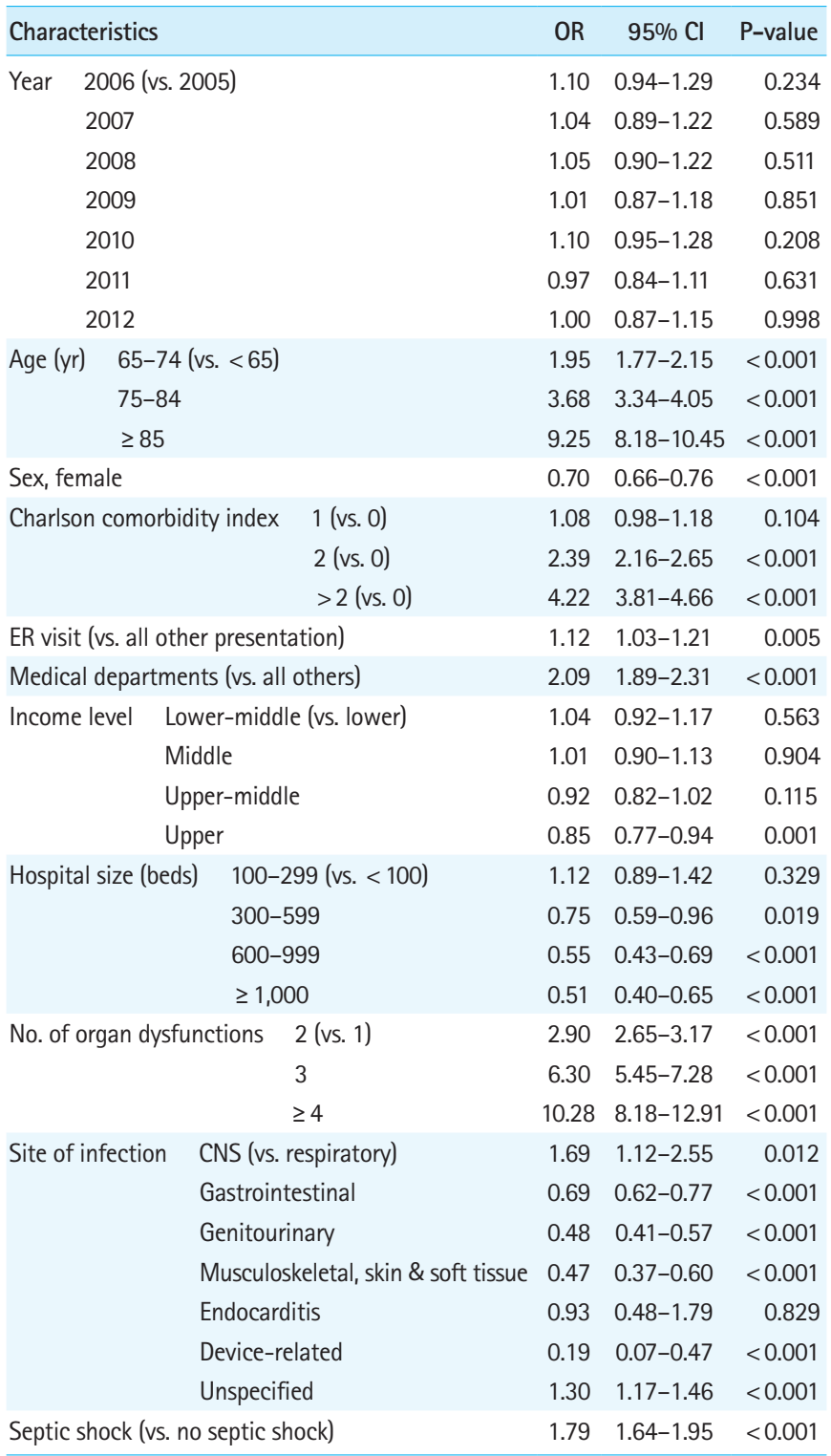

$\mathrm{OR}$, odds ratio; $\mathrm{Cl}$, confidence interval; $\mathrm{ER}$, emergency room; $\mathrm{CNS}$, central nervous system.

overall incidence was increasing and the increase was most prominent in the old age and high comorbidity populations. This rapid increase among old and debilitated sepsis patients was accompanied by the overall increase in mortality. The increase in incidence rate and the cost per case were accompanied by more than a triple increase in national expenditure on sepsis. Considering that population aging is still an ongoing process in Korea, we believe these trends will continue for a while. ${ }^{26}$

We found several interesting but worrisome risk factors for mortality from sepsis. The association between lower family income level and higher mortality can be the sign of disparities in the quality of critical care according to income levels. Although
Table 3. Parsimonious multivariable logistic regression model derived from training set

\begin{tabular}{|c|c|c|c|c|c|c|}
\hline \multicolumn{4}{|c|}{ Characteristics } & $\mathrm{OR}$ & $95 \% \mathrm{Cl}$ & P-value \\
\hline \multirow[t]{3}{*}{ Age (yr) } & \multicolumn{3}{|c|}{$65-74$ (vs. <65) } & 1.91 & $1.71-2.12$ & $<0.001$ \\
\hline & \multicolumn{3}{|c|}{$75-84$} & 3.63 & $3.27-4.02$ & $<0.001$ \\
\hline & \multicolumn{3}{|l|}{$\geq 85$} & 9.16 & $8.01-10.48$ & $<0.001$ \\
\hline \multicolumn{4}{|c|}{ Sex, female } & 0.70 & $0.64-0.76$ & $<0.001$ \\
\hline \multirow{3}{*}{\multicolumn{3}{|c|}{ Charlson comorbidity index }} & 1 (vs. 0) & 1.10 & $1.00-1.21$ & 0.058 \\
\hline & & & 2 (vs. 0) & 2.33 & $2.08-2.62$ & $<0.001$ \\
\hline & & & $>2$ (vs. 0) & 3.98 & $3.56-4.45$ & $<0.001$ \\
\hline \multicolumn{4}{|c|}{ Medical departments (vs. all others) } & 2.12 & $1.90-2.37$ & $<0.001$ \\
\hline \multirow{4}{*}{\multicolumn{2}{|c|}{ Hospital size (beds) }} & \multicolumn{2}{|c|}{ 100-299 (vs. < 100) } & 1.28 & $0.98-1.66$ & 0.070 \\
\hline & & \multicolumn{2}{|c|}{$300-599$} & 0.88 & $0.68-1.15$ & 0.346 \\
\hline & & \multicolumn{2}{|c|}{$600-999$} & 0.66 & $0.51-0.86$ & 0.002 \\
\hline & & \multicolumn{2}{|c|}{$\geq 1,000$} & 0.60 & $0.46-0.79$ & $<0.001$ \\
\hline \multirow{3}{*}{\multicolumn{3}{|c|}{ No. of organ dysfunctions }} & 2 (vs. 1) & 2.87 & $2.60-3.17$ & $<0.001$ \\
\hline & & & 3 & 6.17 & $5.27-7.23$ & $<0.001$ \\
\hline & & & $\geq 4$ & 11.28 & 8.73-14.58 & $<0.001$ \\
\hline \multirow[t]{7}{*}{ Site of i } & fection & \multicolumn{2}{|c|}{ CNS (vs. respiratory) } & 1.89 & $1.20-2.98$ & 0.006 \\
\hline & & \multicolumn{2}{|c|}{ Gastrointestinal } & 0.70 & $0.62-0.78$ & $<0.001$ \\
\hline & & \multicolumn{2}{|c|}{ Genitourinary } & 0.50 & $0.41-0.60$ & $<0.001$ \\
\hline & & \multicolumn{2}{|c|}{ Musculoskeletal, skin \& soft tissue } & 0.44 & $0.34-0.58$ & $<0.001$ \\
\hline & & \multicolumn{2}{|c|}{ Endocarditis } & 0.95 & $0.46-1.95$ & 0.884 \\
\hline & & \multicolumn{2}{|c|}{ Device-related } & 0.23 & $0.09-0.59$ & 0.002 \\
\hline & & \multicolumn{2}{|c|}{ Unspecified } & 1.30 & $1.15-1.47$ & $<0.001$ \\
\hline \multicolumn{4}{|c|}{ Septic shock (vs. no septic shock) } & 1.79 & $1.63-1.97$ & $<0.001$ \\
\hline
\end{tabular}

$\mathrm{OR}$, odds ratio; $\mathrm{Cl}$, confidence interval; $\mathrm{CNS}$, central nervous system.

there was universal healthcare coverage by the National Health Insurance Service in Korea throughout the study period, there could be significant disparities in care as shown in previous studies of cardiovascular and cancer-related mortalities in the Korean population. ${ }^{27-29}$

Another risk factor was smaller hospital capacity. It is possible that smaller hospitals have narrower ranges of available diagnostic tests and limited capacity for prompt source control which requires duty surgeons or interventional radiologists. It is also possible that the availability of critical care specialists and devices for the treatment of severe organ dysfunction (i.e., continuous renal replacement therapy equipment for acute kidney injury) could also be different. However, there has been no surveillance or any official report on the level of sepsis care provided by hospitals in Korea.

In this study, the age-sex-adjusted mortality rate was about $30 \%$. This is consistent with the findings of previous populationbased studies in which the mortality rate of 'severe sepsis' ranged from $26 \%$ to $40 \%{ }^{4,6,9,15,19,30-32}$ However, these are clearly higher than the 10\% mortality rate used to define 'sepsis' in the Sepsis-3 definition paper.' This difference could be due to methodological differences in identifying organ dysfunctions. Organ dysfunctions 

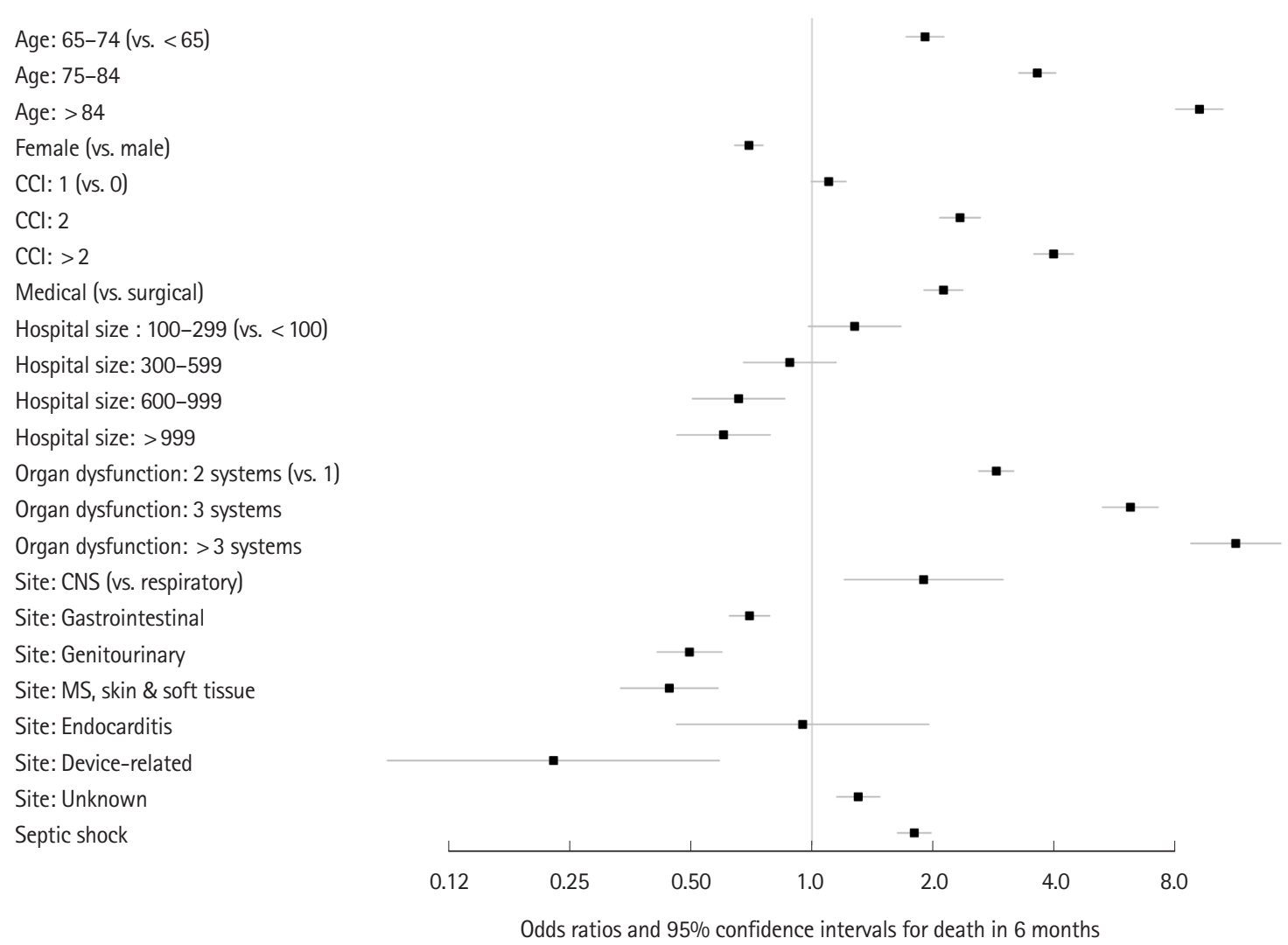

Fig. 5. Predictors of the 6-month mortality model of sepsis. CCl, Charlson comorbidity index; CNS, central nervous system; MS, musculoskeletal.

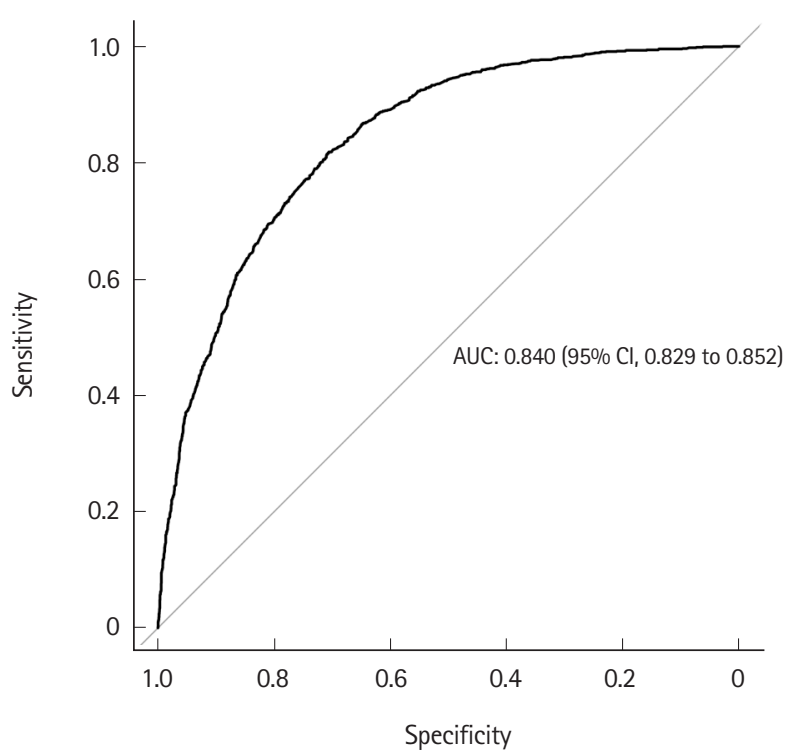

Fig. 6. Predictive performance of the 6-month mortality model of sepsis. AUC, area under the receiver operating characteristic curve; $\mathrm{Cl}$, confidence interval.

in population-based studies are identified based-on diagnostic and intervention codes. Because only significant clinical/laboratory derangements would lead to any entry of related diagnostic codes or application of interventions, organ dysfunctions identified in population-based studies must be more severe.

Lastly, the association between ER presentation and increased mortality could be an important finding. More than half of the patients received their initial care in the ER and their outcomes were worse than the other patients. Currently, we do not know whether this is due to the severity of diseases among ER patients or the quality of care provided in the ERs. However, we believe that the ER should be regarded as a critical intervention point for sepsis care as the ward and ICU have been, because the initial treatment of more than half of sepsis cases would be initiated in the ER and there might be a lot of room for improvement.

The coding scheme used in this study is unique in several aspects. First, the coding system was based on the ICD-10 in contrast to previous studies that were based on the ICD-9. Second, we used a more comprehensive set of diseases for case definition. Third, we limited the cases that were treated with intravenous antibiotics. Fourth, we applied several new operational definitions for organ dysfunction as described in the Appendix 1.

This study has several limitations. First, this study was based on claim data in which the accuracy of diagnostic codes was limited and significant over- or under-coding could be present. Sec- 
ond, it was impossible to accurately know the timing of clinical events. Although the sequence of events based on the order of the fragmented claim records of each admission case was considered, the sequence of events within each claim record was not available. Third, the operational definition used to define sepsis cases was arbitrary and could lead to possible misclassifications. Lastly, the overall cost and mortality rate were all-cause estimates. Therefore, it should be considered that a significant proportion of them would be attributable to non-sepsis conditions.

Despite these limitations, this study, as other population-based studies using claim data, allowed for the examination of health care utilization and expenditure of sepsis in a real-world setting of Korea. For the first time, it estimated the overall burden of sepsis and the overall trend in the epidemiology of sepsis in Korea using nationally representative cohort data. It also identified risk factors for death in sepsis some of which may have implications for our health care delivery system.

In conclusion, sepsis is relatively common and is associated with a high mortality rate in Korea. Although the current national burden of sepsis is already high, it is expected to increase at a fast pace because of the ongoing population aging in Korea. Proactive measures to curtail this increase in societal burden should be sought and implemented.

\section{CONFLICT OF INTEREST}

Kyuseok Kim is an editorial board member of Clinical Experimental and Emergency Medicine; however, he did not involve in the paper reviewer selection, evaluation, and decision process of this article. Otherwise, no potential conflict of interest relevant to this article was reported.

\section{ACKNOWLEDGMENTS}

This study was supported by grant no. 17-2017-026 from the Seoul National University Bundang Hospital research fund. This study was also supported by grant no. 09-2015-001 from the Seoul National University Bundang Hospital research fund.

\section{SUPPLEMENTARY MATERIAL}

Supplementary Fig. 1 is available from: https://doi.org/10.15441/ ceem.18.007.

\section{REFERENCES}

1. Singer M, Deutschman CS, Seymour CW, et al. The third inter- national consensus definitions for sepsis and septic shock (Sepsis-3). JAMA 2016;315:801-10.

2. Vincent JL, Marshall JC, Namendys-Silva SA, et al. Assessment of the worldwide burden of critical illness: the intensive care over nations (ICON) audit. Lancet Respir Med 2014;2:380-6.

3. Rhodes A, Evans LE, Alhazzani W, et al. Surviving sepsis campaign: international guidelines for management of sepsis and septic shock: 2016. Intensive Care Med 2017;43:304-77.

4. Martin GS, Mannino DM, Eaton S, Moss M. The epidemiology of sepsis in the United States from 1979 through 2000. N Engl J Med 2003;348:1546-54.

5. Williams MD, Braun LA, Cooper LM, et al. Hospitalized cancer patients with severe sepsis: analysis of incidence, mortality, and associated costs of care. Crit Care 2004;8:R291-8.

6. Ho BC, Bellomo R, McGain F, et al. The incidence and outcome of septic shock patients in the absence of early-goal directed therapy. Crit Care 2006;10:R80.

7. Esper AM, Moss M, Lewis CA, Nisbet R, Mannino DM, Martin GS. The role of infection and comorbidity: factors that influence disparities in sepsis. Crit Care Med 2006;34:2576-82.

8. Danai PA, Sinha S, Moss M, Haber MJ, Martin GS. Seasonal variation in the epidemiology of sepsis. Crit Care Med 2007; 35:410-5.

9. Dombrovskiy VY, Martin AA, Sunderram J, Paz HL. Rapid increase in hospitalization and mortality rates for severe sepsis in the United States: a trend analysis from 1993 to 2003. Crit Care Med 2007;35:1244-50.

10. Wang HE, Shapiro NI, Angus DC, Yealy DM. National estimates of severe sepsis in United States emergency departments. Crit Care Med 2007;35:1928-36.

11. Barnato AE, Alexander SL, Linde-Zwirble WT, Angus DC. Racial variation in the incidence, care, and outcomes of severe sepsis: analysis of population, patient, and hospital characteristics. Am J Respir Crit Care Med 2008;177:279-84.

12. Vincent JL, Rello J, Marshall J, et al. International study of the prevalence and outcomes of infection in intensive care units. JAMA 2009;302:2323-9.

13. Mayr FB, Yende $S$, Linde-Zwirble WT, et al. Infection rate and acute organ dysfunction risk as explanations for racial differences in severe sepsis. JAMA 2010;303:2495-503.

14. Lagu $T$, Lindenauer $P K$, Rothberg $M B_{\text {, et al. Development and }}$ validation of a model that uses enhanced administrative data to predict mortality in patients with sepsis. Crit Care Med 2011; 39:2425-30.

15. Levy MM, Artigas A, Phillips GS, et al. Outcomes of the Surviving Sepsis Campaign in intensive care units in the USA and Europe: a prospective cohort study. Lancet Infect Dis 2012;12: 
919-24.

16. Herring $A A$, Ginde $A A$, Fahimi J, et al. Increasing critical care admissions from U.S. emergency departments, 2001-2009. Crit Care Med 2013;41:1197-204.

17. Villar J, Clement JP, Stotts J, et al. Many emergency department patients with severe sepsis and septic shock do not meet diagnostic criteria within 3 hours of arrival. Ann Emerg Med 2014;64:48-54.

18. Stevenson EK, Rubenstein AR, Radin GT, Wiener RS, Walkey AJ. Two decades of mortality trends among patients with severe sepsis: a comparative meta-analysis. Crit Care Med 2014; 42:625-31.

19. Filbin MR, Arias SA, Camargo CA Jr, Barche A, Pallin DJ. Sepsis visits and antibiotic utilization in U.S. emergency departments. Crit Care Med 2014;42:528-35.

20. Moore JX, Donnelly JP, Griffin R, Howard G, Safford MM, Wang HE. Defining sepsis mortality clusters in the United States. Crit Care Med 2016:44:1380-7.

21. Lee J, Lee JS, Park SH, Shin SA, Kim K. Cohort profile: the National Health Insurance Service-National Sample Cohort (NHISNSC), South Korea. Int J Epidemiol 2017;46:e15.

22. Quan $H$, Sundararajan $V$, Halfon $P$, et al. Coding algorithms for defining comorbidities in ICD-9-CM and ICD-10 administrative data. Med Care 2005;43:1130-9.

23. Kim KH. Comparative study on three algorithms of the ICD-10 Charlson comorbidity index with myocardial infarction patients. J Prev Med Public Health 2010;43:42-9.

24. Sharabiani MT, Aylin P, Bottle A. Systematic review of comorbidity indices for administrative data. Med Care 2012;50:110918.
25. Statistics Korea. 2010 Korean census [Internet]. Daejeon: Statistics Korea; 2015 [cited 2017 May 10]. Available from: http: //kostat.go.kr/portal/korea/kor_nw/2/2/6/index.board?bmode $=$ readctaSeq $=347102$.

26. Statistics Korea. Future Korean population estimation [Internet]. Daejeon: Statistics Korea; 2016 [cited 2017 May 10]. Available from http://kostat.go.kr/portal/korea/kor_nw/2/1/ index.board bmode $=$ read\&taSeq $=357935$.

27. Song YM, Ferrer RL, Cho SI, Sung J, Ebrahim S, Davey Smith G. Socioeconomic status and cardiovascular disease among men: the Korean national health service prospective cohort study. Am J Public Health 2006;96:152-9.

28. Kim CW, Lee SY, Moon OR. Inequalities in cancer incidence and mortality across income groups and policy implications in South Korea. Public Health 2008;122:229-36.

29. Yim J, Hwang SS, Yoo KY, Kim CY. Contribution of income-related inequality and healthcare utilisation to survival in cancers of the lung, liver, stomach and colon. J Epidemiol Community Health 2012;66:37-40.

30. Angus DC, Linde-Zwirble WT, Lidicker J, Clermont G, Carcillo J, Pinsky MR. Epidemiology of severe sepsis in the United States: analysis of incidence, outcome, and associated costs of care. Crit Care Med 2001;29:1303-10.

31. Shen HN, Lu CL, Yang HH. Epidemiologic trend of severe sepsis in Taiwan from 1997 through 2006. Chest 2010;138:298304.

32. Fleischmann C, Scherag A, Adhikari NK, et al. Assessment of global incidence and mortality of hospital-treated sepsis. current estimates and limitations. Am J Respir Crit Care Med 2016; 193:259-72. 


\section{Appendix 1. Definition of a sepsis case}

Admission cases with 1) bacterial or fungal infection or the conditions that are often complicated by such infections (e.g. surgical abdomen) as indicated by the primary or the first secondary diagnostic codes, 2) prescription of intravenous antibiotics and 3) presence of organ dysfunction involving at least one system.

Coding conventions for both diagnostic and procedure codes:

a) Dot (.) is omitted from diagnostic codes (i.e. J38.3 $\rightarrow$ J383)

b) If there are more than a code in the same hierarchy (i.e. J38 and J383), the parent codes indicate only themselves specifically, but not its children (i.e. J38x).

c) The code at the end of each of hierarchy, including those without any parent, indicate both themselves and their children (i.e. J37x for J37)

\section{1) Korean Classification of Diseases sixth revision codes used for identification of infectious conditions}

A00, Cholera; A01, Typhoid and paratyphoid fevers; A02, Other salmonella infections; A03, Shigellosis; A04, Other bacterial intestinal infections; A05, Other bacterial foodborne intoxications, NEC; A09, Other gastroenteritis and colitis of infectious and unspecified origin; A20, Plague; A21, Tularaemia; A23, Brucellosis; A24, Glanders and Melioidosis; A25, Rat-bite fevers; A26, Erysipeloid; A27, Leptospirosis; A28, Other zoonotic bacterial diseases, NEC; A31, Infection due to other mycobacteria; A32, Listeriosis; A35, Other tetanus; A36, Diphtheria; A37, Whooping cough; A38, Scarlet fever; A39, Meningococcal infection; A40, Streptococcal sepsis; A41, Other sepsis; A42, Actinomycosis; A43, Nocardiosis; A44, Bartonellosis; A46, Erysipelas; A48, Other bacterial diseases, NEC; A49, Bacterial infection of unspecified site; A54, Gonococcal infection; A55, Chlamydial lymphogranuloma (venereum); A56, Other sexually transmitted chlamydial diseases; A57, Chancroid; A58, Granuloma inguinale; A63, Other predominantly sexually transmitted diseases, NEC; A64, Unspecified sexually transmitted disease; A68, Relapsing fevers; A69, Other spirochaetal infections; A70, Chlamydia psittaci infection; A74, Other diseases caused by chlamydiae; A75, Typhus fever; A77, Spotted fever (tick-borne rickettsioses); A78, 0 fever; A79, Other rickettsioses; B37, Candidiasis; B38, Coccidioidomycosis; B39, Histoplasmosis; B40, Blastomycosis; B41, Paracoccidioidomycosis; B42, Sporotrichosis; B43, Chromomycosis and pheomycotic abscess; B44, Aspergillosis; B45, Cryptococcosis; B46, Zygomycosis; B48, Other mycoses, NEC; B49, Unspecified mycosis; B99, Other and unspecified infectious diseases; D71, Progressive septic granulomatosis; D733, Abscess of spleen; D738, Perisplenitis; E060, Abscess of thyroid; E321, Abscess of thymus; G00, Bacterial meningitis, NEC; G01, Meningitis in bacterial diseases classified elsewhere; G02, Meningitis in other infectious and parasitic diseases classified elsewhere; G03, Meningitis due to other and unspecified causes; G04, Encephalitis, myelitis and encephalomyelitis; G05, Encephalitis, myelitis and encephalomyelitis in diseases classified elsewhere; G06, Intracranial and intraspinal abscess and granuloma; G07, Intracranial and intraspinal abscess and granuloma in diseases classified elsewhere; G08, Intracranial and intraspinal phlebitis and thrombophlebitis; H050, Acute inflammation of orbit; H440, Purulent endophthalmitis; H441, Other endophthalmitis; H600, Abscess of external ear; H601, Cellulitis of external ear; H602, Malignant otitis externa; H603, Other infective otitis externa; H608, Other otitis externa; H609, Otitis externa, unspecified; H610, Perichondritis of external ear; H620, Otitis externa in bacterial diseases classified elsewhere; H622, Otitis externa in mycoses; H623, Otitis externa in other infectious and parasitic diseases classified elsewhere; H624, Otitis externa in other diseases classified elsewhere; H66, Suppurative and unspecified otitis media; H67, Otits media in diseases classified elsewhere; H7O, Mastoiditis and related conditions; H750, Mastoiditis in infectious and parasitic diseases classified elsewhere; 130, Acute pericarditis; I32, Pericarditis in diseases classified elsewhere; I33, Acute and subacute endocarditis; I38, Endocarditis, valve unspecified; 139, Endocarditis and heart valve disorders in disease classified elsewhere; 1518, Carditis (acute, chronic); 180, Phlebitis and thrombophlebitis; 1831, Varicose veins of lower extremities with inflammation; 1832, Varicose veins of lower extremities with both ulcer and inflammation; 188, Nonspecific lymphadenitis; 1891, Lymphangitis; J01, Acute sinusitis; J02, Acute pharyngitis; J03, Acute tonsillitis; J04, Acute laryngitis and tracheitis; J05, Acute obstructive laryngitis (croup) and epiglottitis; J13, Pneumonia due to Streptococcus 
pneumoniae; J14, Pneumonia due to Hemophilus in fluenzae; J15, Bacterial pneumonia, NEC; J16, Pneumonia due to other infectious organisms, NEC; J17, Pneumonia in diseases classified elsewhere; J18, Pneumonia, organism unspecified; J20, Acute bronchitis; J21, Acute bronchiolitis; J22, Unspecified acute lower respiratory infection; J340, Abscess, furuncle and carbuncle of nose; J36, Peritonsillar abscess; J383, Abscess of vocal cord(s); J387, Abscess of larynx; J390, Retropharyngeal and parapharyngeal abscess; J391, Other abscess of pharynx; J440, Chronic obstructive pulmonary disease with acute lower respiratory infection; J441, Chronic obstructive pulmonary disease with acute exacerbation, unspecified; J690, Pneumonitis due to food and vomit; J85, Abscess of lung and mediastinum; J86, Pyothorax; J90, Pleurisy with effusion; J91, Pleural effusion in conditions classified eleswhere; J950, Sepsis of tracheostomy stoma; J985, Mediastinitis; J986, Diaphragmatitis; K044, Acute apical periodontitis of pulpal origin; K046, Periapical abscess with sinus; K047, Periapical abscess without sinus; K050, Acute gingivitis; K052, Acute periodontitis; K102, Inflammatory conditions of jaws; K103, Alveolitis of jaws; K112, Sialoadenitis; K113, Abscess of salivary gland; K122, Cellulitis and abscess of mouth; K140, Abscess of tongue; K221, Fungal ulcer of oesophagus; K223, Perforation of oesophagus; K251, Acute gastric ulcer with perforation; K252, Acute gastric ulcer with both hemorrhage and perforation; K255, Chronic or unspecified gastric ulcer with perforation; K256, Chronic or unspecified gastric ulcer with both hemorrhage and perforation; K261, Acute duodenal ulcer with perforation; K262, Acute duodenal ulcer with both hemorrhage and perforation; K265, Chronic or unspecified duodenal ulcer with perforation; K266, Chronic or unspecified duodenal ulcer with both hemorrhage and perforation; K271, Acute peptic ulcer, site unspecified with perforation; K272, Acute peptic ulcer, site unspecified with both hemorrhage and perforation; K275, Chronic or unspecified peptic ulcer, site unspecified with perforation; K276, Chronic or unspecified peptic ulcer, site unspecified with both hemorrhage and perforation; K281, Acute gastrojejunal ulcer with perforation; K282, Acute gastrojejunal ulcer with both hemorrhage and perforation; K285, Chronic or unspecified gastrojejunal ulcer with perforation; K286, Chronic or unspecified gastrojejunal ulcer with both hemorrhage and perforation; K35, Acute appendicitis; K36, Other appendicitis; K37, Unspecified appendicitis; K383, Fistula of appendix; K388, Intussusception of appendix; K401, Bilateral inguinal hernia, with gangrene; K404, Unilateral or unspecified inguinal hernia, with gangrene; K411, Bilateral femoral hernia, with gangrene; $K 414$, Unilateral or unspecified femoral hernia, with gangrene; K421, Umbilical hernia with gangrene; K431, Ventral hernia with gangrene; K441, Diaphragmatic hernia with gangrene; K451, Other specified abdominal hernia with gangrene; K461, Unspecified abdominal hernia with gangrene; K550, Acute vascular disorders of intestine; K551, Chronic vascular disorders of intestine; K559, Vascular disorders of intestine, unspecified; K561, Intussusception; K562, Volvulus; K563, Gallstone ileus; K564, Other impaction of intestine; K565, Intestine adhesions (bands) with obstruction; K566, Other and unspecified intestinal obstruction; K567, Ileus, unspecified; K57, Diverticulitis of intestine (small, large); K60, Fissure and fistula of anal and rectal regions; K61, Abscess of anal and rectal regions; K630, Abscess of intestine; K631, Perforation of intestine (nontraumatic); K632, Fistula of intestine; K65, Peritonitis; K67, Disorders of peritoneum in infectious diseases classified elsewhere; K75, Other inflammatory liver diseases; K770, Liver disorders in infectious and parasitic diseases classified elsewhere; K800, Calculus of gallbladder with acute cholecystitis; K801, Calculus of gallbladder with other cholecystitis; K803, Calculus of bile duct with cholangitis; K804, Calculus of bile duct with cholecystitis; K81, Cholecystitis; K820, Obstruction of gallbladder; K822, Perforation of gallbladder; K823, Fistula of gallbladder; K830, Cholangitis; K831, Obstruction of bile duct; K832, Perforation of bile duct; K833, Fistula of bile duct; K85, Acute pancreatitis; K868, Pancreatic necrosis NOS; K931, Megacolon in Chagasi`s disease (B57.3+); L00, Staphylococcal scalded skin syndrome; L01, Impetigo; L02, Cutaneous abscess, furuncle and carbuncle; L03, Cellulitis; L04, Acute lymphadenitis; L050, Pilonidal cyst with abscess; L08, Other local infections of skin and subcutaneous tissue; M00, Pyogenic arthritis; M01, Direct infections of joint in infectious and parasitic diseases classified elsewhere; M462, Osteomyelitis of vertebra; M463, Infection of intervertebral disc (pyogenic); M464, Discitis, unspecified; M465, Other infective spondylopathies; M491, Brucella spondylitis (A23.-+); M492, Enterobacterial spondylitis (A01-A04+); M493, Spondylopathy in other infectious and parasitic diseases classified elsewhere; M600, Infective myositis; M630, Myositis in bacterial diseases classified elsewhere; M632, Myositis in other infectious diseases classified elsewhere; M650, Abscess of tendon sheath; M651, Other infective (teno) synovitis; M680, Synovitis and tenosynovitis in bacterial diseases classified elsewhere; M710, Abscess of bursa; M711, Other infective bursitis; M715, Other bursitis NEC; M719, Bursitis NOS; M726, Necrotising fasciitis; M728, Abscess of fascia; M730, Gonococcal bursitis (A54.4+); M86, Osteomyelitis; M901, Periostitis in other infectious diseases classified elsewhere; M902, Osteopathy in other infectious diseases classified elsewhere; N10, Acute pyelonephritis; N12, Pyelonephritis NOS; N136, Pyonephrosis; N151, Renal and perinephric abscess; N159, Infection of kidney, NOS; N2O, Calculous pyelonephritis; N390, Urinary tract infection, site not specified; N41, Inflammatory diseases of prostate; N431, Infected hydrocele; N45, Orchitis and epididymitis; N482, Other inflammatory disorders of penis; N49, Inflammatory disorders of male genital organs, NEC; N510, Gonococcal prostatitis (A54.2+); N511, Chlamydial epididymitis (A56.1+); N512, Balanitis in diseases classified elsewhere; N61, Inflammatory disorders of breast; N70, Salpingitis and oophoritis; N71, Inflammatory disease of uterus, except cervix; N72, Inflammatory 
disease of cervix uteri; N73, Other female pelvic inflammatory diseases; N74, Female pelvic inflammatory disorders in diseases classified elsewhere; N751, Abscess of Bartholini's gland; N758, Bartholinitis; N76, Other inflammation of vagina and vulva; N77, Vulvovaginal ulceration and inflammation in diseases classified elsewhere; N82, Fistulae involving female genital tract; N980, Infection associated with artificial insemination; R02, Gangrene, NEC; R091, Pleurisy; R50, Fever of other and unknown origin; R572, Septic shock; R578, Endotoxic shock; R65, Systemic Inflammatory Response Syndrome; T793, Post-traumatic wound infection, NEC; T801, Phlebitis following infusion, transfusion and therapeutic injection; T802, Infections following infusion, transfusion and therapeutic injection; T814, Infection following a procedure, NEC; T826, Infection and inflammatory reaction due to cardiac valve prosthesis; T827, Infection and inflammatory reaction due to other cardiac and vascular devices, implants and grafts; T835, Infection and inflammatory reaction due to prosthetic device, implant and graft in urinary system; T836, Infection and inflammatory reaction due to prosthetic device, implant and graft in genital tract; T845, Infection and inflammatory reaction due to internal joint prosthesis; T846, Infection and inflammatory reaction due to internal fixation device (any site); T847, Infection and inflammatory reaction due to other internal orthopedic prosthetic devices, implants and grafts; T857, Infection and inflammatory reaction due to other internal prosthetic devices,implants and grafts; T874, Infection of amputation stump; T880, Infection following immunization; U80, Agent resistant to penicillin and related antibiotics; U81, Agent resistant to vancomycin and related antibiotics; U88, Agent resistant to multiple antibiotics; U89, Agent resistant to other and unspecified antibiotics

\section{2) Intravenous antibiotics (generic/product codes for intravenous form was used for actual case definition, not included in the appendix)}

amikacin, amoxicillin, amphotericin, ampicillin, anidulafungin, arbekacin, astromycin, azithromycin, aztreonam, caspofungin, cefamandole, cefazolin, cefbuperazone, cefepime, cefmenoxime, cefmetazole, cefminox, cefodizime, cefonicid, cefoperazone, cefotaxime, cefotetan, cefotiam, cefoxitin, cefpiramide, cefpirome, cefsulodin, ceftazidime, ceftezole, ceftizoxime, ceftriaxone, cefuroxime, cephalothin, cephazedone, cephradine, chloramphenicol, ciprofloxacin, clarithromycin, clindamycin, colistin, doripenem, ertapenem, flomoxef, fluconazole, fosfomycin, gemifloxacin, gentamicin, imipenem, isepamicin, itraconazole, kanamycin, levofloxacin, lincomycin, linezolid, meropenem, metronidazole, micafungin, moxifloxacin, nafcillin, netilmicin, ofloxacin, ornidazole, panipenem, penicillin, pentamidine, piperacillin, ribostamycin, sisomicin, spectinomycin, streptomycin, sulfamethoxazole, teicoplanin, ticarcillin, tigecycline, tobramycin, vancomycin, voriconazole

\section{3) Types of organ dysfunction}

\begin{tabular}{lll}
\hline Types of organ dysfunction & Codes & \\
\hline Hemodynamic & R572 & Septic shock \\
195 & Hypotension \\
1958 & Other hypotension \\
& 1959 & Hypotension, unspecified \\
R57 & Shock, NEC \\
R578 & Other shock \\
R579 & Shock, unspecified \\
T811 & Shock (endotoxic, hypovolemic) during or following a procedure \\
Extra \#1 & Use of vasopressor (norepinephrine, dopamine, dobutamine, vasopressin, epinephrine) \\
J80 & Adult respiratory distress syndrome \\
J81 & Pulmonary edema \\
J96 & Respiratory failure, NEC \\
J960 & Acute respiratory failure \\
J969 & Respiratory failure, unspecified \\
R0902 & Hypoxemia \\
R230 & Cyanosis \\
Z991 & Dependence on respirator \\
&
\end{tabular}


Continued

\begin{tabular}{|c|c|c|}
\hline Types of organ dysfunction & Codes & Conditions \\
\hline & Extra \#1 & $\begin{array}{l}\text { Combination of oxygen delivery, arterial blood gas analysis and lower respiratory infection } \\
\text { (A15, A16, A202, A212, A221, A241, A310, A37, A420, A430, A481, A70, B371, B380, B381, } \\
\text { B382, B390, B391, B392, B400, B401, B402, B410, B420, B440, B441, B450, B460, J13, } \\
\text { J14, J15, J16, J17, J18, J20, J21, J22, J40, J440, J441, J690, J85, J86, J90, J91, R091) }\end{array}$ \\
\hline & Extra \#2 & Mechanical ventilation (M5830, M585) without general anesthesia (L010, L050, L6, L7) \\
\hline \multirow[t]{10}{*}{ Neurologic } & F05 & Delirium, not induced by alcohol and other psyhoactive substances \\
\hline & F06 & Other mental disorders due to brain damage and dysfunction and to physical disease \\
\hline & F09 & Organic psychosis NOS \\
\hline & G931 & Anoxic brain damage, NEC \\
\hline & G934 & Encephalopathy, unspecified \\
\hline & G9380 & Metabolic encephalopathy \\
\hline & R40 & Somnolence, stupor and coma \\
\hline & R400 & Somnolence \\
\hline & R401 & Stupor \\
\hline & R410 & Disorientation, unspecified \\
\hline \multirow[t]{9}{*}{ Hematologic } & D65 & Disseminated intravascular coagulation (defibrination syndrome) \\
\hline & D68 & Other coagulation defects \\
\hline & D688 & Other specified coagulation defects \\
\hline & D689 & Coagulation defect, unspecified \\
\hline & D69 & Purpura and other hemorrhagic conditions \\
\hline & D695 & Secondary thrombocytopenia \\
\hline & D696 & Thrombocytopenia, unspecified \\
\hline & R233 & Spontaneous ecchymoses \\
\hline & R791 & abnormal coagulation lab \\
\hline \multirow[t]{4}{*}{ Hepatic } & K72 & Hepatic failure, NEC \\
\hline & K762 & Central hemorrhagic necrosis of liver \\
\hline & K763 & Infarction of liver \\
\hline & R17 & Unspecified jaundice \\
\hline \multirow[t]{7}{*}{ Renal } & N17 & Acute renal failure \\
\hline & N19 & Unspecified renal failure \\
\hline & N990 & Postprocedural renal failure \\
\hline & R34 & Anuria and oliguria \\
\hline & R944 & Abnormal results of kidney function studies \\
\hline & Z992 & Dependence on renal dialysis \\
\hline & Extra \#1 & $\begin{array}{l}\text { New initiation of hemodialysis / continuous renal replacement therapy }(0701,0702,0703 \text {, } \\
0704,0705) \text { without preexisting dependence on HD (registered kidney disability grade } 1)\end{array}$ \\
\hline \multirow{4}{*}{$\begin{array}{l}\text { Sepsis-induced } \\
\text { cardiomyopathy }\end{array}$} & 1428 & Other cardiomyopathies \\
\hline & 1429 & Cardiomyopathy, unspecified \\
\hline & 1430 & Cardiomyopathy in infectious and parasitic diseases classified elsewhere \\
\hline & 15181 & Takotsubo syndrome (Stress induced cardiomyopathy) \\
\hline Metabolic & E872 & Acidosis \\
\hline
\end{tabular}




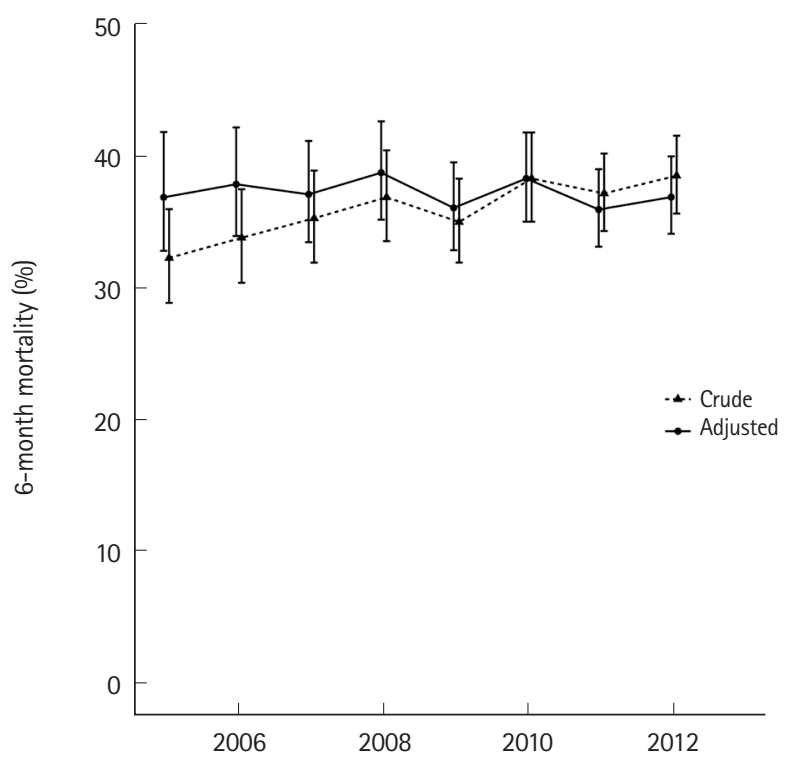

Supplementary Fig. 1. Eight-year trend of sepsis mortality in patients with septic shock. 